\title{
Solitary extramedullary plasmacytoma presenting as an adrenal tumor: case report and literature review
}

\author{
Umar Zeb Khan ${ }^{1}$, Matiullah Masroor ${ }^{2}$, Wenlong Yang ${ }^{1}$, Muhammad Riaz ${ }^{3}$, Hai Liu ${ }^{1}$ \\ ${ }^{1}$ Department of General Surgery, The Third Xiangya Hospital of Central South University, Changsha, China; ${ }^{2}$ Department of General Surgery, \\ The Second Xiangya Hospital of Central South University, Changsha, China; ${ }^{3}$ Department of General Surgery, Affiliated Hospital of North Sichuan \\ Medical College, Nanchong, China \\ Correspondence to: Hai Liu. Department of General Surgery, The Third Xiangya Hospital of Central South University, 138 Tongzipo Rd., Changsha \\ 410013, China. Email: minisurgery@126.com.
}

\begin{abstract}
Solitary extramedullary plasmacytoma, an extremely rare case which accounts for about $3 \%$ of all plasma cell neoplasms, often occurs in the head and neck region such as para nasal sinuses, nasal cavity and oral cavity, it also occurs in the lymph nodes, lungs, thyroid, intestine, liver, pancreas, testis, breast, or skin. Isolated primary plasmacytoma of adrenal is extremely rare and only nine documented cases have been reported in the literature. We are presenting the $10^{\text {th }}$ case which is the youngest patient until now with symptoms of fever and hepatosplenomegaly. A 19-year-old female was admitted with an irregular fever for 20 days. After a series of investigations were carried out there were no CRAB symptoms (hypercalcemia, renal failure, anemia and bone lesions), no free light chain and no more than $10 \%$ increase in plasma cell on bone marrow examination. Computed tomography (CT) scan revealed a tumor in the left adrenal region, and it was diagnosed to be a solitary extramedullary plasmacytoma on biopsy. She underwent the tumor resection one month after admission and recovered well after operation without fever and was discharged from hospital on the thirteenth post-operative day. She has been followed up for 5 years without any sign and symptom of tumor recurrence. Extramedullary plasmacytoma of adrenal gland in an extremely rare disease and usually diagnosed late in life but it can present in younger patients with variable symptoms. However, the surgical treatment yields excellent long-term results. So, complete surgical resection of the lesion is not only a good diagnostic measure, but also an intent-for-cure treatment for solitary adrenal extramedullary plasmacytoma.
\end{abstract}

Keywords: Solitary extramedullary plasmacytoma (SEP); adrenal neoplasm; solitary adrenal extramedullary plasmacytoma

Submitted Oct 22, 2020. Accepted for publication Jan 14, 2021.

doi: $10.21037 /$ gs-20-773

View this article at: http://dx.doi.org/10.21037/gs-20-773

\section{Introduction}

Plasma cell dyscrasias accounts for about $10 \%$ of all hematological neoplasms (1). According to 2017 WHO classification for plasma cell neoplasms (PCN), it includes non-IgM monoclonal gammopathy of undetermined significance (MGUS), plasma cell myeloma (PCM), plasmacytoma, monoclonal immunoglobulin deposition disease and PCN with associated paraneoplastic syndrome. Plasmacytoma is further subdivided into solitary plasmacytoma of bone (SPB) and extramedullary plasmacytoma (2). The age standardized incidence rate of multiple myeloma is 2.1 per 100,000 person and death rate is 1.5 per 100,000 persons globally in 2016 which increase from 1990 to 2016 by $126 \%$ and $94 \%$ respectively (3). Solitary extramedullary plasmacytoma (SEP) is a very rare disease and only accounts for about $3 \%$ of all plasma cell malignancies (4). It often occurs in the head and neck region, such as the paranasal sinuses, nasal cavities, and oral cavity (1), it also occurs in the gastrointestinal tract $(5,6)$, pancreas (7), liver (8) parotid gland (9), respiratory 
system (10), central nervous system (11), thyroid (12), lymph nodes (13), breast (14), testes (15), retroperitoneum (16), or skin (17) etc. Before being diagnosed, it is necessary to perform relevant examinations to ascertain whether there is Bence-Jonce protein in the blood and urine, osteolytic lesions in spine and pelvis, renal insufficiency and hypercalcemia, to completely rule out multiple myeloma $(1,18)$. Primary solitary lesions in the adrenal region are most unusual. We report a case of primary SEP of the adrenal gland and briefly review the literature. We present the following case in accordance with the CARE reporting checklist (available at http://dx.doi. org/10.21037/gs-20-773).

\section{Case presentation}

A 19-year-old female with no past medical, surgical and family history with irregular fever for more than 20 days was admitted to our hospital. The patient had been treated with antibiotics at a local hospital and had no improvement in symptoms. Since onset, her appetite, sleep, defecation and urination are normal but she lost about 10 kilograms of weight. She was then referred to a tertiary hospital, where she was treated with antibiotics such as cefotaxime and moxifloxacin, but her symptoms did not improve. Bone marrow biopsy showed normal hematopoiesis with no more than $10 \%$ increase in plasma cells. Complete blood cell count: white blood cell (WBC) $13.08 \times 10^{9} / \mathrm{L}$, neutrophil $68.7 \%$, platelet count (PLT) $483 \times 10^{12} / \mathrm{L}$, hemoglobin (HGB) $65 \mathrm{~g} / \mathrm{L}$; the full set of viral tests were negative except herpes simplex virus (HSV) IgG which was positive; antibodies to mycoplasma pneumoniae, chlamydia and legionella were all negative; vaginal discharge was positive for trichomonas; tests of sex hormones, adrenal hormones and thyroid function were generally normal. In liver function test other indexes were within the normal range except low albumin and high globulin. Pregnancy test was negative; serum immunoglobulin assay: IgG $27.1 \mathrm{~g} / \mathrm{L}$ (normal 5.16$14.26 \mathrm{~g} / \mathrm{L}$ ), IgA $4.57 \mathrm{~g} / \mathrm{L}$ (normal $0.80-5.00 \mathrm{~g} / \mathrm{L}$ ), IgM $2.35 \mathrm{~g} / \mathrm{L}$ (normal $0.30-2.09 \mathrm{~g} / \mathrm{L}$ ), IgE $41.25 \mathrm{IU} / \mathrm{mL}$ (normal 0-358.00 IU/L), complement-3 and complement-4 was in normal limit; serum $\lambda$ chain $13.3 \mathrm{~g} / \mathrm{L}, \kappa$ chain $23.0 \mathrm{~g} / \mathrm{L}$; Erythrocyte sedimentation rate was greater than $140 \mathrm{~mm} / \mathrm{h}$.

Physical examination on admission: body temperature $37.5^{\circ} \mathrm{C}$, pulse 130 beats per minute, blood pressure $118 / 75 \mathrm{mmHg}$. She was anemic in appearance with liver and spleen palpable just under the costal edge of abdomen without tenderness. No jaundice, lymphadenopathy and no added breath sounds on bilateral auscultation. Nerve reflexes were normal.

After admission, relevant laboratory tests were conducted and the results were as follows: blood and urine cultures for bacterial and blood culture for fungal infection were negative; hypersensitive C-reactive protein was $16.4 \mathrm{mg} /$ $\mathrm{L}$ (normal $<5 \mathrm{mg} / \mathrm{L}$ ), lactate dehydrogenase was normal; HGB electrophoresis was normal and Combs test was negative. Bence-Jonce protein was negative in both urine and serum; tumor markers were negative; adrenal hormones were within normal limit; urine kappa light chain was $0.1 \mathrm{mg} / \mathrm{L}$ (normal $3.3-19.4 \mathrm{mg} / \mathrm{L}$ ), lambda light chain was $0.43 \mathrm{mg} / \mathrm{L}$ (normal $5.70-26.30 \mathrm{mg} / \mathrm{L}$ ); serum immunoglobulin assay: IgA $3.18 \mathrm{~g} / \mathrm{L}$ (normal 0.80 $5.00 \mathrm{~g} / \mathrm{L}$ ), IgE $34.00 \mathrm{IU} / \mathrm{mL}$ (normal 0-358.00 IU/L), IgG $23.40 \mathrm{~g} / \mathrm{L}$ (normal 5.16-14.26 g/L), IgM $2.81 \mathrm{~g} / \mathrm{L}$ (normal 0.30-2.09 g/L), complements were normal; tests for connective tissue disease were negative; the second bone marrow biopsy showed normal hematopoiesis with no more than $10 \%$ increase in plasma cells. The distribution of WBCs in peripheral blood was normal, and the size of mature red blood cells was inconsistent; platelets are distributed in clusters. Related tests for B-cell lymphoma, including Ig gene rearrangement test, MYD 88 gene $\mathrm{L} 265 \mathrm{P}$ mutation test and $b c-2 / 7 H$ gene rearrangement test, were all negative.

After admission, symptomatic treatment was given, and cefoperazone $1 \mathrm{~g}$ IV 12 hourly and meropenem $1 \mathrm{~g}$ IV 8 hourly were successively used, but the irregular fever was still uncontrolled, with the maximum temperature reaching $40^{\circ} \mathrm{C}$. On the 20 th day of admission, $40 \mathrm{mg}$ methylprednisolone was used per day, and on third day the fever was controlled therefore, the methylprednisolone discontinued, and oral prednisolone $20 \mathrm{mg}$ daily for 5 days started. Antibiotics were discontinued on day 27 of admission. Computed tomography (CT) scan revealed an isolated mass in the left adrenal region: a solitary mass in the left adrenal region with few tiny calcifications on the medial margin of the tumor (Figure 1A); the density of the lesions was not uniform (Figure $1 B$ ), the value of plain CT was about 28-42 HU, the value of arterial CT scan was about 33-65 HU, portal was about 33-79 HU, delayed was about 33-83 HU. Coronal section CT of abdomen shows a well circumscribed tumor in the left adrenal region with no osteolytic destruction of vertebrae and pelvis and Hepatosplenomegaly (Figure 2); then, a CT-guided biopsy of the mass was performed on the $7^{\text {th }}$ 

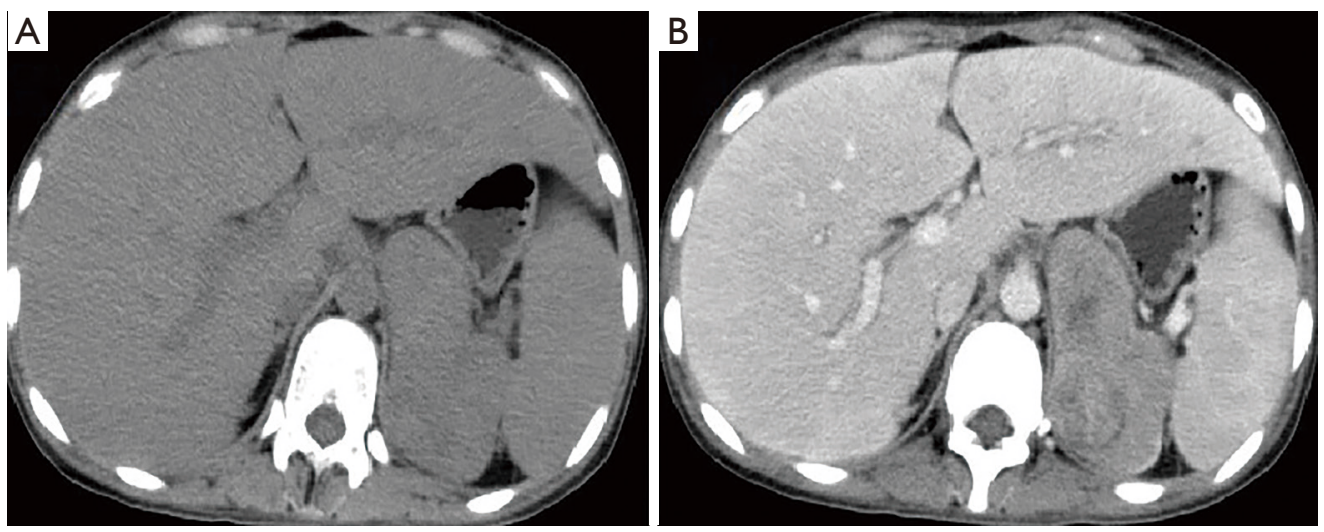

Figure 1 CT cross-sectional scan showed a well-circumscribed adrenal tumor. (A) Plain scan showed tiny calcification at the medial margin of tumor; (B) contrast enhanced scan showed heterogenous tumor density. CT, computed tomography.

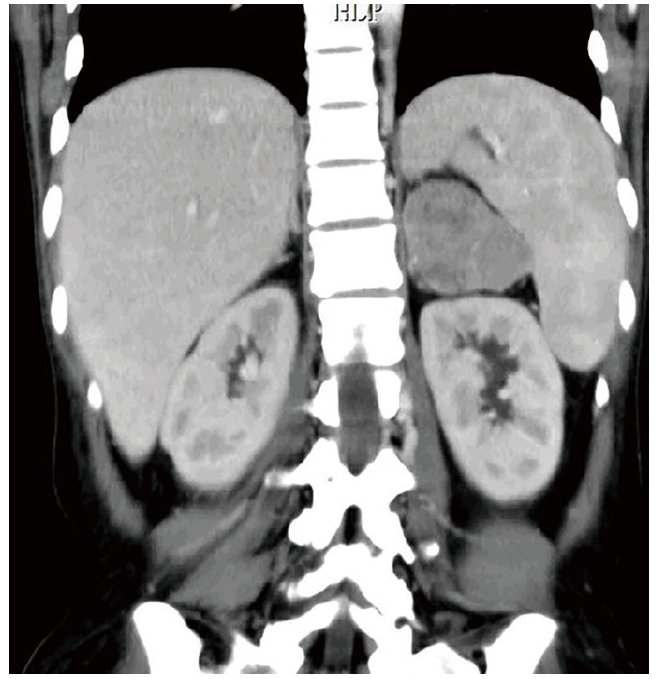

Figure 2 CT coronal scan showed a well-circumscribed tumor in the left adrenal region, no osteolytic destruction of the vertebrae and pelvis. CT, computed tomography.

day of admission. Pathological diagnosis of needle biopsy was reported later as follows: round cell tumor or tumorlike lesion, mainly plasma cell hyperplasia, combined with immunohistochemical analysis, the tumor was considered as lymphocyte hematopoietic system tumor, and extramedullary plasmacytoma can be considered after further examination to exclude heavy chain disease and immunoglobulin related diseases. So, the primary diagnosis was adrenal extramedullary plasmacytoma and surgical excision of the lesion was recommended after multidisciplinary consultation. In order to remove the tumor and further confirm the diagnosis, the patient and his family agreed to undergo surgery.

On the 33rd day of admission she underwent excision of tumor, because the patient's liver and spleen were enlarged, it was difficult to undergo laparoscopic surgery, so traditional laparotomy was adopted. An L-shaped surgical incision was made in the left upper abdomen, and surgical exploration revealed hepatosplenomegaly, enlarged left hepatic segments and spleen occupying the left upper abdomen with extremely less free abdominal space, leading to the difficulty for the operation. The tumor was found to be located inwardly above the left kidney, posterior to the pancreas, and the upper pole of the tumor reached posterior to the gastric fundus. The tumor was lobulated, hard, immovable, and about $8 \mathrm{~cm} \times 6 \mathrm{~cm} \times 5 \mathrm{~cm}$ in size. The left coronary ligament of the liver was first cut off with an electrocautery to free the left liver and lifted it up outside the surgical incision to expose the stomach and spleen. The gastrocolic ligament and the retroperitoneal folds above pancreas were cut with an electrocautery, and then the posterior peritoneum of the upper margin of the pancreatic body was incised to expose the splenic artery. Then the splenic artery was temporarily ligated with silk thread to make the spleen shrink, and therefore to get more operating space. This manipulation not only made more free room for surgery, but also prevented the dangerous massive hemorrhage from splenic vessels. Then, the splenophrenic ligaments, splenocolic ligaments and lienorenal ligaments were successively cut off with the help of electrocautery and the pancreatic body with tail and spleen could be separated from post abdominal wall and transferred to the outside of 

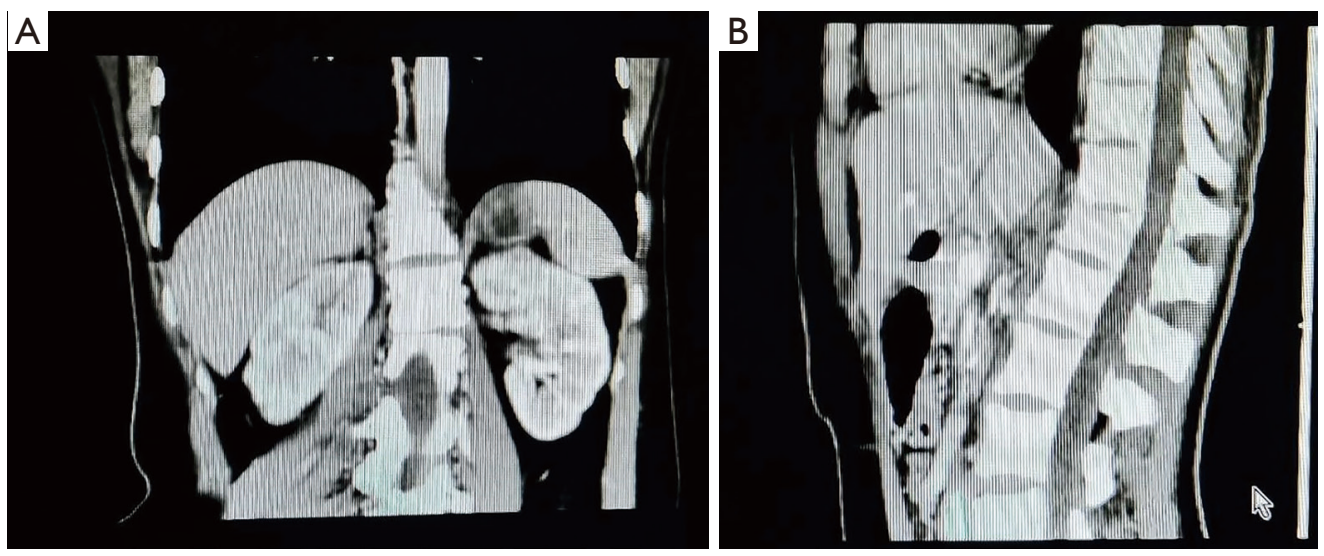

Figure 3 A follow-up CT scan 5 years after surgery. (A) A coronal section showing normal liver, spleen and kidneys without recurrence of adrenal tumor; (B) a sagittal section showing no osteolytic lesions of bone. CT, computed tomography.

the body through the surgical incision, and at this point the mass was completely exposed in front of us. The tumor had a clear boundary with pseudo capsule and was closely related to the left adrenal gland. The whole tumor with part of the adrenal tissue was excised. Hemostasis of tumor bed was achieved with 3/0 proline continuous suture. After the surgical wound was examined and the bleeding stopped completely, the spleen and liver were returned to the abdominal cavity and restored to the normal anatomical position. The operation was successful and the bleeding was less than $200 \mathrm{~mL}$.

The pathological examination of the surgical specimen showed that the gross appearance of tumor capsule was intact, and the necrotic area was visible inside; adrenal tissue is seen around the tumor; in the background of lymphocyte and plasma cell, the distribution of large cells with round shape and polygon shape could be seen; combined with other relevant examinations and immunohistochemical staining, the pathological diagnosis was considered as plasmacytoma; immunohistochemical staining: CD38 (-), CD138 (+), Kappa (+), Lambda (-), IgA (+++), IgG (+++), $\operatorname{IgM}(+)$ and $\mathrm{Ki} 67(15 \%+)$.

She recovered well after the operation with no fever. Gastrointestinal peristalsis returned to normal on the third post-operative day (POD), and she was discharged on the 13th POD after full recovery. After discharge from the hospital, she did not take any other adjuvant treatment. Regular 6 monthly follow-up showed normal complete blood count, liver and kidney function, no osteolytic lesions and no evidence of tumor recurrence. As of this writing, five years have passed since the operation and she is in excellent health. CT scan of the last follow up given in (Figure 3).
All procedures performed in studies involving human participants were in accordance with the ethical standards of the institutional and/or national research committee(s) and with the Helsinki Declaration (as revised in 2013). Written informed consent was obtained from the patient.

\section{Discussion}

Most patients with plasmacytoma are diagnosed with a systemic disease called multiple myeloma. SEP is very rare and accounts only for about $3 \%$ of all PCN (4). Although SEP may occur throughout the body, the majority of cases occur in the head and neck region, particularly in the upper airways, including the nasal cavity, sinuses, oropharynx, salivary glands, and larynx, it also can be seen in gastrointestinal tract, pancreas, liver, parotid gland, respiratory system, central nervous system, thyroid, lymph nodes, mammary gland, testes, retroperitoneum or skin $(1,5-17)$. The diagnosis of SEP requires the presence of extramedullary tumor infiltrated with clonal plasma cells and bone marrow biopsy without any clonal plasma cell in the absence of any multiple myeloma symptoms $(1,18)$. SEP of adrenal is extremely rare and only nine documented cases have been reported in the literature. The case described here is the $10^{\text {th }}$ case of SEP with the youngest patient ever presented in literature who presented with an isolated adrenal mass with no evidence of myeloma elsewhere, including no monoclonal protein in serum or urine, no plasma cell tumor-associated hypercalcemia, renal impairment, or multiple osteolytic lesions. Compared with the case reported in the literature, this case has some unique 


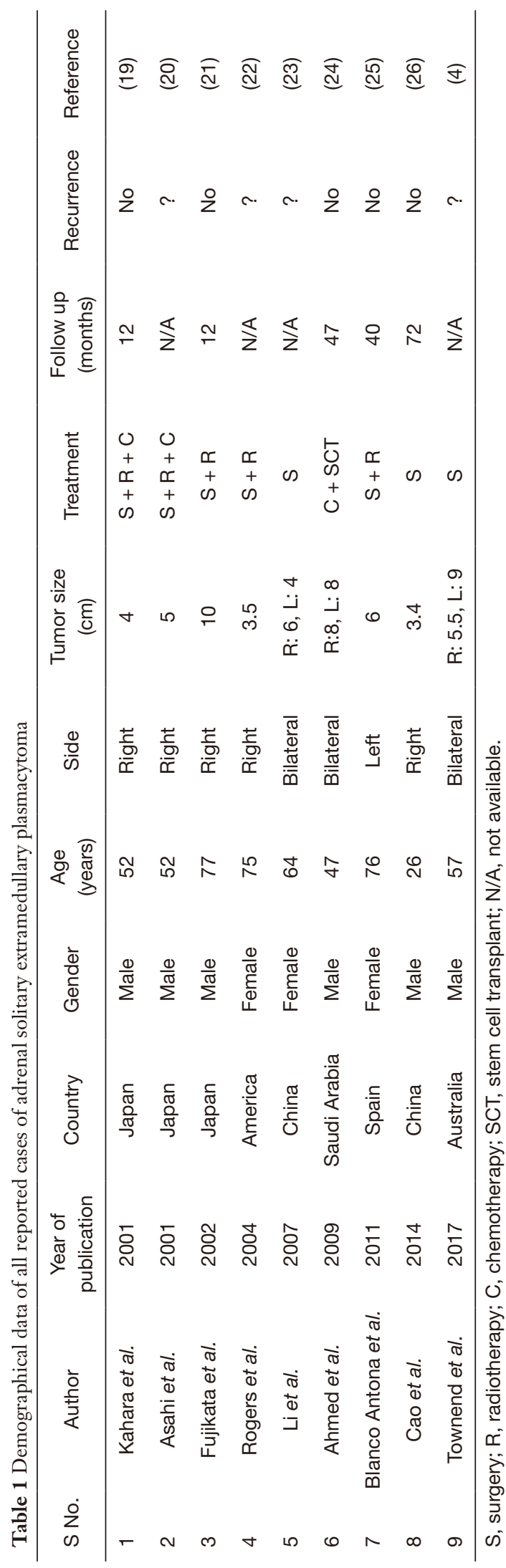

clinical features, namely, she is the youngest, with a long history of irregular fever, anemia, and hepatosplenomegaly. The causes of anemia and hepatosplenomegaly are unknown and may be related to long-term fever. The nine cases reported in literature are shown in Table 1 which are either asymptomatic and diagnosed incidentally or with variable symptoms like back pain, flank pain, muscle pain, hip pain, fatigue or abdominal cramping (4,19-26). By the time this manuscript was written, she had been followed up for 5 years, and all of her symptoms had returned to normal. She was currently in excellent physical condition.

Since most SEP occur in the head and neck, curative surgery is usually a mutilating procedure for tumors located in these areas, so radiation therapy should be used instead of surgery (27). However, there are no clear guidelines for the treatment of SEP located elsewhere such as in adrenal region, which have been treated with different modalities, including, surgical resection combined with radiotherapy or chemotherapy, chemotherapy with stem cell transplant and surgery alone as shown in Table 1 . If boundary is clear, the tumor can be removed by radical surgery and that may be the best treatment. The cases reported in literature eight out of nine has been treated with surgery in which three cases treated only with surgery other 3 treated with surgery plus radiotherapy and the rest of 2 treated with surgery combined with radiotherapy and chemotherapy. The longest follow up has been reported for a patient treated only with surgery by Cao et al. who followed the case for 72 months without the recurrence of disease (26). Therefore, surgical resection should be the first choice for the treatment of adrenal SEP. Surgery is not only beneficial in diagnosing but is also a definitive cure for the disease. If the patient has undergone complete surgery, adjuvant radiation therapy is only required if the surgical margin is inadequate (28). Although the prognosis of SEP is much better than that of SPB, a small number of patients may have local recurrences or develop multiple myeloma after surgery, so patients should be told to review regularly every 3 to 6 months. The follow-up may include urine and serum gel electrophoresis of proteins, M-protein, complete blood count, serum creatinine, and serum calcium. In imaging diagnosis, ultrasound, CT scan and magnetic resonance imaging (MRI) are used to examine the primary lesion site and skeletal system (4).

\section{Conclusions}

Extramedullary plasmacytoma of adrenal is an ultra- 
rare disease which usually diagnosed late in life but it can develop in younger patients, either asymptomatic or with variable sign and symptoms. Radical surgical resection is not only a means of obtaining a definitive diagnosis, but also a promising treatment option. Surgery may allow patients to achieve long-term cancer-free survival.

\section{Acknowledgments}

We would like to thank Dr. Tiebin Jiang, Department of hematology, The Third Xiangya Hospital, Central South University, for her help in the treatment of the case and writing of this manuscript.

Funding: None.

\section{Footnote}

Reporting Checklist: The authors have completed the CARE reporting checklist. Available at http://dx.doi.org/10.21037/ gs-20-773

Peer Review File: Available at http://dx.doi.org/10.21037/gs20-773

Conflicts of Interest: All authors have completed the ICMJE uniform disclosure form (available at http://dx.doi. org/10.21037/gs-20-773). The authors have no conflicts of interest to declare.

Ethical Statement: The authors are accountable for all aspects of the work in ensuring that questions related to the accuracy or integrity of any part of the work are appropriately investigated and resolved. All procedures performed in studies involving human participants were in accordance with the ethical standards of the institutional and/or national research committee(s) and with the Helsinki Declaration (as revised in 2013). Written informed consent was obtained from the patient.

Open Access Statement: This is an Open Access article distributed in accordance with the Creative Commons Attribution-NonCommercial-NoDerivs 4.0 International License (CC BY-NC-ND 4.0), which permits the noncommercial replication and distribution of the article with the strict proviso that no changes or edits are made and the original work is properly cited (including links to both the formal publication through the relevant DOI and the license). See: https://creativecommons.org/licenses/by-nc-nd/4.0/.

\section{References}

1. Basavaiah SH, Lobo FD, Philipose CS, et al. Clinicopathological spectrum of solitary Plasmacytoma: a single center experience from coastal India. BMC Cancer 2019;19:801.

2. Hatem K, Ceren D, Salman F, et al. Plasma Cell Cancer. StatPearls Publishing, 2020.

3. Cowan AJ, Allen C, Barac A, et al. Global Burden of Multiple Myeloma: A Systematic Analysis for the Global Burden of Disease Study 2016. JAMA Oncol 2018;4:1221-7.

4. Townend PJ, Kraus G, Coyle L, et al. Bilateral extramedullary adrenal plasmacytoma: case report and review of the literature. Int J Endocr Oncol 2017;4:67-73.

5. Zhang D, Cao D, Shen D, et al. Extramedullary plasmacytoma occuring in ileocecum: A case report and literature review. Medicine 2017;96:e9313.

6. Glasbey JC, Arshad F, Almond LM, et al. Gastrointestinal manifestations of extramedullary plasmacytoma: a narrative review and illustrative case reports. Ann R Coll Surg Engl 2018;100:371-6.

7. Le M, Surapaneni BK, Jain V, et al. Pancreatic Extramedullary Plasmacytoma Presenting as a Pancreatic Mass. Clin Med Insights Gastroenterol 2018;11:1179552218801603.

8. Lee JY, Won JH, Kim HJ, et al. Solitary extramedullary plasmacytoma of the liver without systemic monoclonal gammopathy. J Korean Med Sci 2007;22:754-7.

9. Abrar S, Ali N, Qureshi BM, et al. Extramedullary plasmacytoma: rare neoplasm of parotid gland. BMJ Case Rep 2018;2018:bcr2017222367.

10. LeNoir B, Bruner ET, Denlinger CE, et al. Extramedullary Plasmacytoma of the Right Main Bronchus. Ann Thorac Surg 2019;108:e119-20.

11. Sidlo J, Sidlova H. Sudden and unexpected death due to intracranial sellar extramedullary plasmacytoma. J Forensic Leg Med 2019;61:89-91.

12. Hassan MJ, Khans S, Pujani M, et al. Extramedullary plasmacytoma of the thyroid: report of a rare case. Blood Res 2014;49:280-3.

13. Naymagon L, Abdul-Hay M. Primary extramedullary plasmacytoma with diffuse lymph node involvement: a case report and review of the literature. J Med Case Rep 2019;13:153.

14. Proctor NS, Rippey JJ, Shulman G, et al. Extramedullary plasmacytoma of the breast. J Pathol 1975;116:97-100.

15. Shimokihara K, Kawahara T, Chiba S, et al. Extramedullary plasmacytoma of the testis: A case report. 
Urol Case Rep 2017;16:101-3.

16. Oh D, Kim CK, Park BK, et al. Primary extramedullary plasmacytoma in retroperitoneum: CT and integrated PET/CT findings. European Journal of Radiology Extra 2007;62:57-61.

17. Lim CC, Soong TK, Chuah KC, et al. Extramedullary plasmacytoma of the eyelid: a case report and review of literature. Clin Exp Optom 2013;96:349-51.

18. Lai CY, Hsieh HH, Chen HK, et al. Clinical Features of Head and Neck Solitary Extramedullary Plasmacytoma in Taiwan. In Vivo 2020;34:261-5.

19. Kahara T, Nagai Y, Yamashita H, et al. Extramedullary plasmacytoma in the adrenal incidentaloma. Clin Endocrinol (Oxf) 2001;55:267-70.

20. Asahi H, Iwasa Y, Komatsu K, et al. A case of plasmacytoma involving adrenal gland. Hinyokika Kiyo 2001;47:629-31.

21. Fujikata S, Tanji N, Aoki K, et al. Extramedullary plasmacytoma arising from an adrenal gland. Urology 2002;60:514.

22. Rogers CG, Pinto PA, Weir EG. Extraosseous (extramedullary) plasmacytoma of the adrenal gland. Arch

Cite this article as: Khan UZ, Masroor M, Yang W, Riaz M, Liu H. Solitary extramedullary plasmacytoma presenting as an adrenal tumor: case report and literature review. Gland Surg 2021;10(3):1158-1164. doi: 10.21037/gs-20-773
Pathol Lab Med 2004;128:e86-8.

23. Li Y, Guo YK, Yang ZG, et al. Extramedullary plasmacytoma involving the bilateral adrenal glands on MR imaging. Korean J Radiol 2007;8:246-8.

24. Ahmed M, Al-Ghamdi A, Al-Omari M, et al. Autologous bone marrow transplanation for extramedullary plasmacytoma presenting as adrenal incidentaloma. Ann Saudi Med 2009;29:219-22.

25. Blanco Antona F, Bahamonde Cabria S, Blanco Antona L, et al. Adrenal extramedullary plasmacytoma. Cir Esp 2011;89:690-1.

26. Cao D, Li L, Liu L, et al. Solitary extramedullary plasmacytoma of the adrenal gland: a rare case report with review of the literature. Int J Clin Exp Pathol 2014;7:9072-5.

27. Pham A, Mahindra A. Solitary Plasmacytoma: a Review of Diagnosis and Management. Curr Hematol Malig Rep 2019;14:63-9.

28. Kilciksiz S, Karakoyun-Celik O, Agaoglu FY, et al. A review for solitary plasmacytoma of bone and extramedullary plasmacytoma. ScientificWorldJournal 2012;2012:895765. 